\title{
Pulmonary effect of laryngeal mask airway compare to tracheal tube during the early postoperative period in lower leg vascular surgery patient
}

\author{
Ju Hwan Lee \\ Department of Anesthesiology and Pain Medicine, Wonkwang University School of Medicine, Iksan, Korea
}

Table 1. Dermographic and Hemodynamic Data

\begin{tabular}{lllll} 
& & TT group & LMA group & P \\
\hline Age $(\mathrm{yr})$ & & $64.44 \pm 9.3$ & $64.64 \pm 11.0$ & 0.611 \\
Sex $(\mathrm{M} / \mathrm{F})$ & $52 / 48$ & $48 / 52$ & 0.521 \\
Weight $(\mathrm{kg})$ & & $60.72 \pm 12$ & $62.44 \pm 13$ & 0.421 \\
Height $(\mathrm{cm})$ & & $161.64 \pm 6.1$ & $160.84 \pm 7.1$ & 0.723 \\
Heart rate (beats/ min) & Awake & $66.16 \pm 17$ & $66.20 \pm 16$ & 0.989 \\
& After intubation & $85.80 \pm 19$ & $68.84 \pm 15$ & $0.001^{*}$ \\
& After extubation & $84.04 \pm 16$ & $69.76 \pm 16$ & $0.001^{*}$ \\
Mean arterial pressure (mm & Awake & $80.32 \pm 15$ & $78.96 \pm 16$ & 0.442 \\
Hg) & After intubation & $88.60 \pm 21$ & $79.76 \pm 18$ & $0.001^{*}$ \\
& After extubation & $88.12 \pm 19$ & $80.32 \pm 18$ & $0.001^{*}$ \\
Mean duration of surgery & & $118.34 \pm 22$ & $120.12 \pm 20$ & 0.934 \\
\hline
\end{tabular}

Table 3. Comparison of Postoperative Coughing and Hoarseness in Patients after the Removal of Airway Devices at 60 Minutes

\begin{tabular}{llcl}
\hline & \multicolumn{2}{c}{ No. of patient } & P value \\
\cline { 2 - 3 } & LMA & TT & \\
\hline $\begin{array}{l}\text { Coughing } \\
60 \text { min }\end{array}$ & 0 & 5 & 0.047 \\
$\begin{array}{l}\text { Hoarseness } \\
60 \text { min }\end{array}$ & 2 & 7 & 0.267 \\
\hline
\end{tabular}

Table 4. Comparison of Postoperative

Pulmonary Complication

Table 2. Changes between the Pre- and postoperative Data

\begin{tabular}{lllllllll}
\hline & \multicolumn{2}{l}{ TT group } & \multicolumn{7}{c}{ LMA group } \\
& Pre & post & change & P & pre & post & change & P \\
\hline FVC (L) & 2.85 & 2.46 & $-13.68 \%$ & $0.00^{*}$ & 2.52 & 2.53 & $0.39 \%$ & 0.12 \\
FEV1 (L) & 2.13 & 1.82 & $-14.55 \%$ & $0.00^{*}$ & 1.92 & 1.91 & $-0.52 \%$ & 0.72 \\
FEV1/FVC (\%) & 74.16 & 73.96 & $-0.26 \%$ & 0.76 & 74.76 & 74.68 & $-0.10 \%$ & 0.74 \\
PEFR (L/min) & 335.2 & 284.5 & $-15.12 \%$ & $0.00^{*}$ & 321.6 & 302.1 & $-6.06 \%$ & 0.08 \\
SpO2 (\%) & 98.52 & 97.44 & $-1.09 \%$ & $0.03 *$ & 97.92 & 98.56 & $0.65 \%$ & 0.10
\end{tabular}
during a Weak after Surgery

LMA: laryngeal mask airway; TT: tracheal tube; FVC: forced vital capacity; FEV1: forced expiratory volume in 1st second of expiration; PEFR: peak expiratory flow rate; SPO2: oxygen saturation ${ }^{*} \mathrm{p}<0.05$ in two groups

\section{Background:}

Patients undergoing lower extrimities vascular surgery (eg, bypass and endarterectomy) have a high incidence of coexisting disease, including diabetes mellitus, hypertension, renal impairment, pulmonary disease and old age.

The tracheal tube (TT) produces reversible bronchoconstriction and increases pulmonary airway resistance and wide hemodynamic change compared to the laryngeal mask airway (LMA). This trend can have an effect in pulmonary function during the postoperative period specially vascular surgery patient. The aim of this study was to compare early pulmonary effect in patients undergoing lower leg vascular surgery with the LMA or with the TT.

\section{Methods:}

Sixty patients scheduled for lower leg vascular surgery under general anesthesia were randomized to receive the LMA or the TT. The groups were as follows: (1) LMA group $(\mathrm{n}=30)$ : appropriate size classic LMA was inserted using conventional technique; and (2) TT group $(\mathrm{n}=30)$ : appropriate size cuffed TT was inserted after laryngoscopy. All patients pulmonary functions test data were blindedly recorded Before anesthesia and 1 hour after tube removal, pulse oximetry values ( $\mathrm{SpO} 2)$ were recorded and patients performed forced spirometry in the supine position. And pneumonia and atelectasis was recorded during a weak after operation.

\section{Results:}

The two study groups were comparable with regard to their demographic profiles and ASA physical statuses. The mean duration of surgery was $118.34 \mathrm{~min}$ in the TT group and $120.12 \mathrm{~min}$ in the LMA group (Table 1). Airway device was placed easily in first trail in all patients, and no laryngospasm was noted in any patient.

In the TT group, compared to patients using the LMA, there was a greater relative increase of heart rate, mean arterial pressure (Table 1). There were no differences between groups in the preoperative respiratory function test, and $\mathrm{SpO} 2$. Following surgery comparison of the mean depression of PFTs $1 \mathrm{hr}$ after the removal of device showed significant decreases $\mathrm{SpO}$, forced expiratory volume in the first second (FEV1), forced vital capacity (FVC) and peak expiratory flow (PEFR) in TT groups (Table 2). The FEV1/FVC, did not change in either of the groups (Table 2). The comparison of coughing and hoarseness showed a significantly lower incidence of coughing in LMA group (Table 3). In TT group 2 case of Atelectasis and 1 case of pneumonia noted during a weak after surgery (Table 4).

\section{Conclusions:}

The use of LMA instead of TT for airway management during lower leg vascular surgeries causes less depression of pulmonary functions during the early postoperative period. 